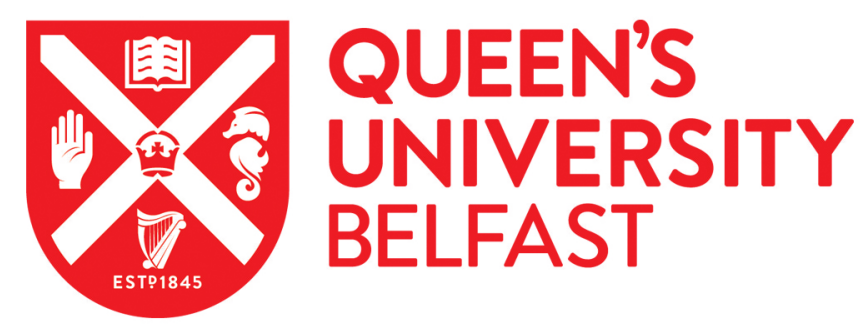

\title{
Using Intrinsic Surfaces to Calculate the Free-Energy Change When Nanoparticles Adsorb on Membranes
}

Klug, J., Triguero, C., Del Popolo, M., \& Tribello, G. (2018). Using Intrinsic Surfaces to Calculate the FreeEnergy Change When Nanoparticles Adsorb on Membranes. Journal of Physical Chemistry B, 1-21.

https://doi.org/10.1021/acs.jpcb.8b03661

Published in:

Journal of Physical Chemistry B

Document Version:

Peer reviewed version

Queen's University Belfast - Research Portal:

Link to publication record in Queen's University Belfast Research Portal

Publisher rights

Copyright $\odot 2018$ American Chemical Society. This work is made available online in accordance with the publisher's policies. Please refer to any applicable terms of use of the publisher.

\section{General rights}

Copyright for the publications made accessible via the Queen's University Belfast Research Portal is retained by the author(s) and / or other copyright owners and it is a condition of accessing these publications that users recognise and abide by the legal requirements associated with these rights.

Take down policy

The Research Portal is Queen's institutional repository that provides access to Queen's research output. Every effort has been made to ensure that content in the Research Portal does not infringe any person's rights, or applicable UK laws. If you discover content in the Research Portal that you believe breaches copyright or violates any law, please contact openaccess@qub.ac.uk. 


\title{
Using Intrinsic Surfaces to Calculate the
}

\section{Free-energy Change When Nanoparticles Adsorb}

\section{on Membranes}

\author{
Joaquín Klug, ${ }^{\dagger, \ddagger}$ Carles Triguero, ${ }^{\dagger}$ Mario G. Del Pópolo, ${ }^{\dagger, \ddagger}$ and Gareth A. \\ Tribello*,† \\ †Atomistic Simulation Centre, School of Mathematics and Physics, Queen's University \\ Belfast, Belfast, BTr7 1NN, United Kingdom \\ $\ddagger$ CONICET and Facultad de Ciencias Exactas y Naturales, Universidad Nacional de Cuyo, \\ Padre Jorge Contreras 1300, CP5500, Mendoza, Argentina \\ E-mail: g.tribello@qub.ac.uk
}




\begin{abstract}
A reaction coordinate that can be used when investigating binding to dynamical surfaces with molecular dynamics is introduced. This coordinate measures the distance between the adsorbate and an isocontour in a density field. Furthermore, the coordinate is continuous so simulation biases that are a function of this coordinate can be added to the Hamiltonian in order to increase the rate of adsorption/desorption. The efficacy of this new coordinates is demonstrated by performing metadynamics simulations to measure the strength with which a hydrophilic nanoparticle binds to a lipid bilayer. An investigation of this binding mechanism that is performed using the coordinate demonstrates that the structure of the lipid bilayer undergoes a series of concerted changes in structure as the nanoparticle binds.
\end{abstract}

\title{
Graphical TOC Entry
}

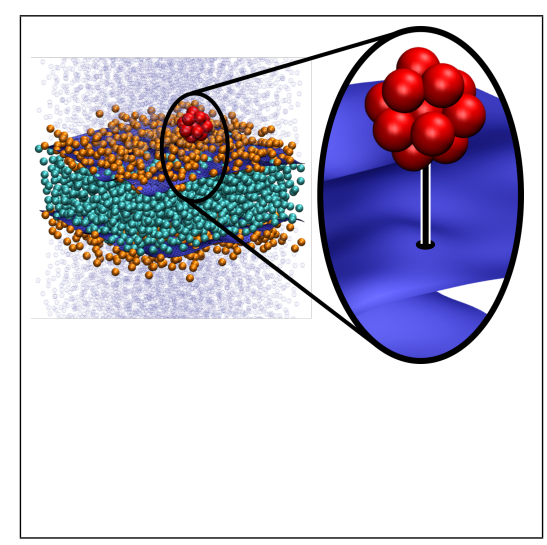


The binding and insertion of solutes into biomembranes has important consequences for biological processes such as cellular uptake, transport, ${ }^{1}$ molecular recognition, ${ }^{2}$ and cellular stress response. ${ }^{3}$ The flux of matter across cell membranes is normally mediated by specific ligand-receptor interactions. Biomembranes are thus commonly in contact with a variety of solutes ranging from ions and small molecules ${ }^{4,5}$ all the way up to biological macromolecules such as proteins and polymeric sugars, however, and many of these molecules will cross the membrane via osmotic processes. Furthermore, there has been much recent interest in how cell membranes interact with the nanoparticles, ${ }^{6-9}$ that are increasingly being used in commercial products ${ }^{10,11}$ and new health technologies. ${ }^{12-16}$ In all these cases, solutemembrane interactions are paramount when it comes to determining the integrity of the membrane and its permeability.

Many simulation studies have probed the mechanism by which drug molecules cross the cell membrane,,$^{4,7,8,17-25}$ as this process has important consequences in terms of drug delivery and design. ${ }^{4,26}$ The effective interaction between the drug and the membrane is normally quantified by measuring the binding free energy profile, which provides information on the thermodynamic cost for bringing the solute from the solution up to a point in the proximity or inside the lipid bilayer. One problem with this approach is the way the distance between the drug and the membrane is evaluated. Typically the distance between the centre of mass of the molecule and the centre of mass of the membrane is used. ${ }^{8,19,20,23,27-31}$ This is problematic ${ }^{32-36}$ however, as the shape and structure of the membrane can change dynamically. In particular, lipid bilayers can undergo shape fluctuations that are caused by long-wavelength capillary waves, bending, curvature or even changes in volume. Furthermore, deviations of these sorts are often enhanced when the membrane is perturbed by a bulky solute. The reason this is problematic is that all of these effects serve to bend the membrane and thus make the distance between the centre of mass of the solute and centre of mass of the membrane, $z_{\text {com }}$, a poor reaction coordinate.

Willard and Chandler ${ }^{37,38}$ proposed a potential solution to this problem in the context of 
studying the evaporation of water. They introduced a coarse-grained representation of the atomic density, and used the distance between the evaporating water molecule and an isocontour in this three dimensional density as a collective variable (CV) for measuring how far the evaporating water molecule is from the liquid surface. Willard and Chandler's method has since been used to study a range of liquid-liquid interfaces. ${ }^{38,39}$ Furthermore, many researchers have used techniques similar to Willard and Chandler's to extract the properties of a membrane from a simulation trajectory. ${ }^{40-42}$ To our knowledge, however, no one has applied this technique to study membrane-solute interactions and no one has used it in tandem with metadynamics. In the present paper we thus use Willard and Chandler's method to examine the binding between a nanoparticle and a fluid lipid bilayer. Metadynamics simulations are performed to extract the nanoparticle binding free energy curve, and to investigate how the adsorption of the nanoparticle affects the shape and thickness of the membrane. We show that the new CV effectively resolves the problems of dealing with the dynamic interface of the membrane that were encountered in previous works, and that we can thus rapidly extract reliable estimates for the free energy of binding by making use of it.

Willard and Chander's method ${ }^{37,38}$ for finding the position of the water surface starts by introducing an instantaneous density field for water molecules:

$$
\rho(x, y, z)=\sum_{i=1}^{N} K\left(\frac{x-x_{i}}{\lambda}, \frac{y-y_{i}}{\lambda}, \frac{z-z_{i}}{\lambda}\right)
$$

In our work the sum in this expression runs over the atoms in the molecules that make up the membrane. Each of these atoms is located at a position $\left(x_{i}, y_{i}, z_{i}\right)$ and the $K$ is a smooth, 3-dimensional Kernel function (e.g. a Gaussian) that integrates to one. Each atom in the membrane thus contributes a function that is peaked at the atom's position and which decays over some characteristic length scale $\lambda$ to the final field $\rho(x, y, z)$. The value of $\rho(x, y, z)$ is thus large if this function is evaluated at a point that is inside the membrane and small if it is evaluated at some position outside the membrane. Consequently, we can find interfaces that 
separate the membrane from the surrounding solution by finding the 2-dimensional manifold containing points for which:

$$
\varphi(x, y, z)-\varphi_{0}=0
$$

In this expression $\varphi_{0}$ is a parameter and $\varphi(x, y, z)$ is calculated using equation 1 . As shown in the first two panels of figure 1 this procedures converts a particle based representation of the membrane and surrounding solution into a coarse grained representation that describes the extents of the membrane and aqueous phases and the location of the interface between them.

In this work we are interested in studying the binding of a nanoparticle to a lipid bilayer. We thus do not need to calculate the full Willard-Chandler surface and can instead simply compute the perpendicular distance between the nanoparticle and the membrane surface as illustrated in the second panel of figure 1 . In other words, if the nanoparticle is at $\left(x_{0}, y_{0}, z_{0}\right)$ and if we choose to set up the cell so that the membrane surface is perpendicular to the $z$ axis, we can evaluate $\left(z_{s}-z_{0}\right)$ with $z_{s}$ being the value of $z$ that satisfies:

$$
\varphi\left(x_{0}, y_{0}, z_{s}\right)-\varphi_{0}=0
$$

A potential problem with this approach is illustrated in the middle panel of figure 1 . As this figure shows there are generally two points along the $z$ axis which satisfy this condition. One of these points lies on the top surface of the membrane, while the other lies on the bottom surface. The manner in which we resolved this problem is illustrated in the third panel of figure 1 , however. We find the two $z$ values that satisfy equation $3, z_{s}^{(1)}$ and $z_{s}^{(2)}$, and then evaluate the following collective variable:

$$
s=\left(z_{0}-z_{s}^{(1)}\right)\left(z_{0}-z_{s}^{(2)}\right)
$$

As shown in the final panel of figure 1 the value of this $\mathrm{CV}$ is thus positive if the $\mathrm{CV}$ is outside of the membrane envelope and negative if it is inside the membrane. Furthermore, 

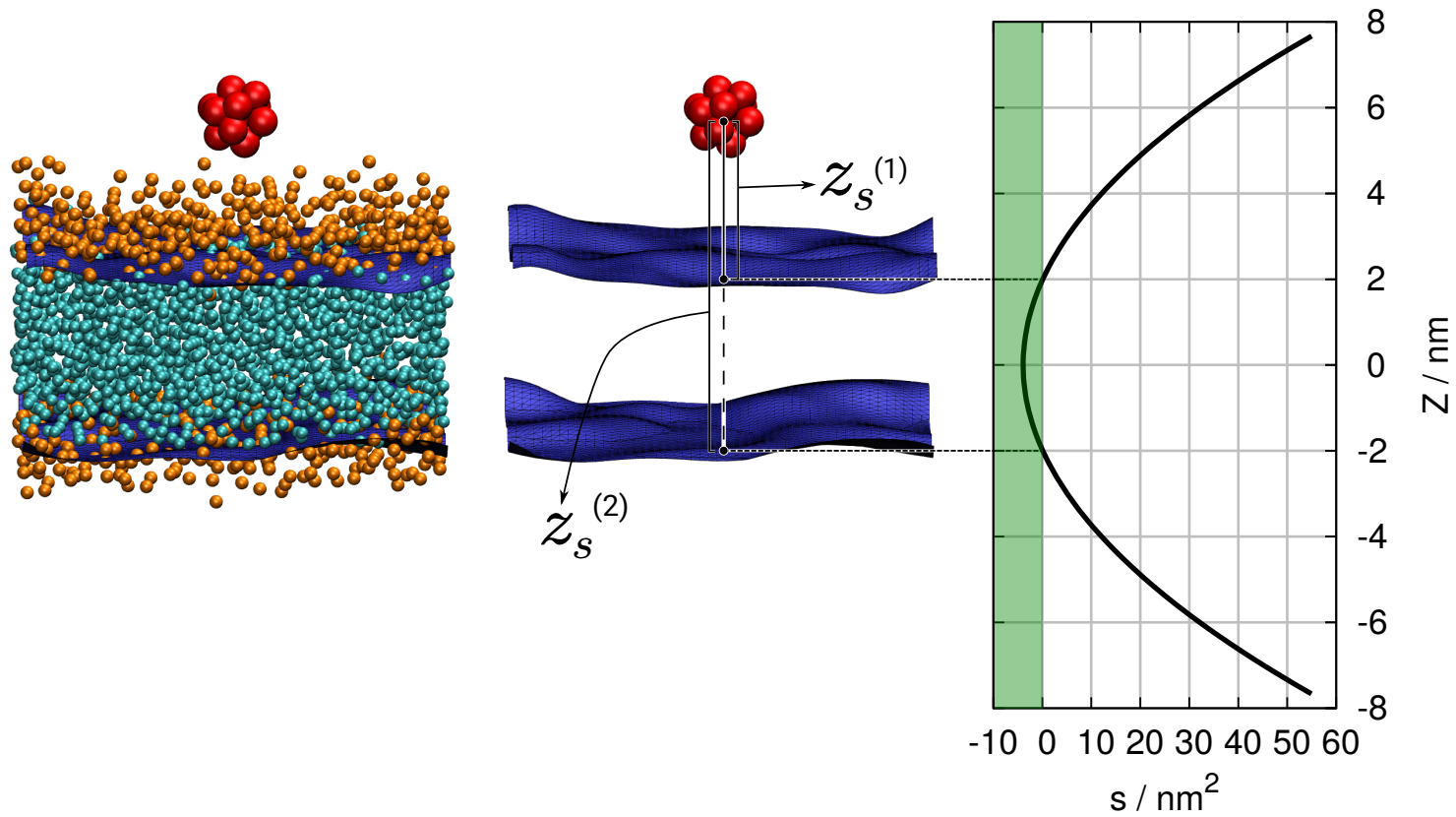

Figure 1: An illustration showing how the distance from countour CV that we have introduced in this work is defined. The left panel shows a snapshot of the simulated system. The atoms in the nanoparticle are in red, the lipid heads are in orange and the lipid tails are in cyan. The two intrinsic-surfaces, which we use to describe the spatial extent of the membrane, are shown in blue. These surfaces are shown again in the central panel, together with lines that indicate how the distances from the closest and other surfaces of the membrane, $z_{s}^{(1)}$ and $z_{s}^{(2)}$, that appear in equation 4 are measured. The graph in the right panel shows how the final CV, $s=\left(z_{0}-z_{s}^{(1)}\right)\left(z_{0}-z_{s}^{(2)}\right)$, depends on the $Z$-coordinate of the nanoparticle. The green shading is used to indicate that this $\mathrm{CV}$ is negative when the particle is inside the membrane and positive when the particle is outside the membrane.

the value of the CV and its derivatives are all continuous and can thus be used in free energy calculations. We thus implemented this new CV and its derivatives in PLUMED 2.3. ${ }^{43}$

The final problem to resolve is related to the periodic boundaries of the simulations cell. When the nanoparticle moves far from either membrane surfaces the value of $s$ becomes negative, even though it is not inside the membrane envelope. The reason for this spurious behaviour is that when the nanoparticle is far from the membrane one of the solutions of equation 3 corresponds to the position of the membrane surface in one of the periodic replicas. This problem is easily solved, however, by adding a wall on the values of $s$ that prevents the nanoparticle from diffusing too far from the membrane surface.

To test the collective variable outlined in the previous paragraphs, we ran metadynam- 


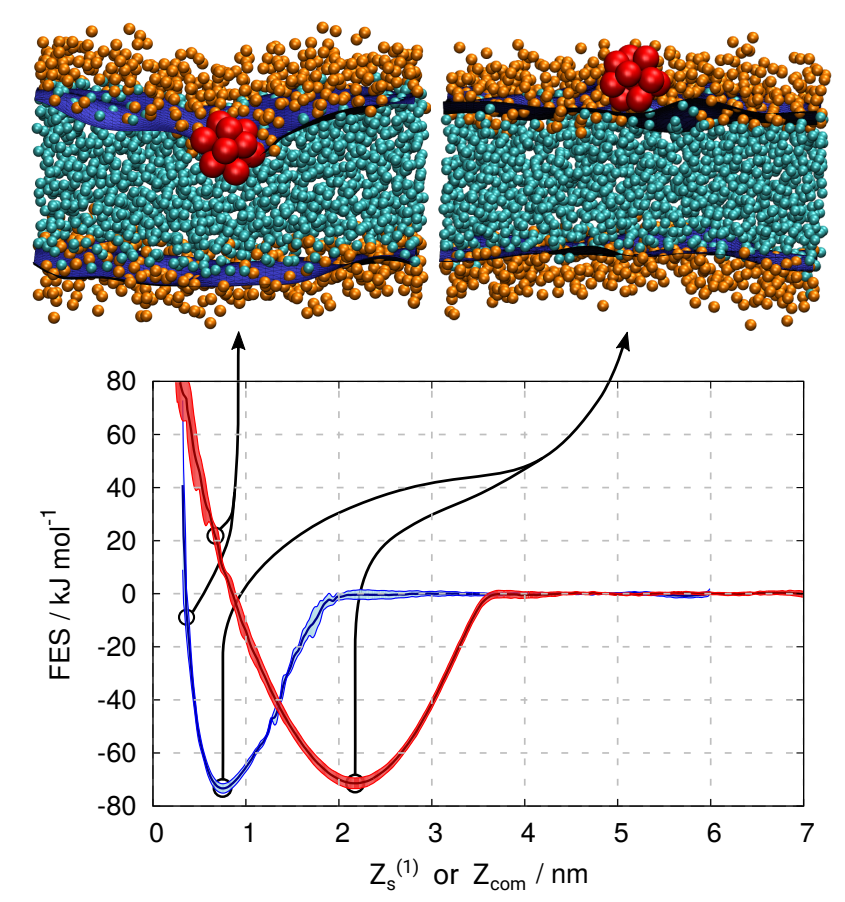

Figure 2: Free energy as a function of the distance between the center of the nanoparticle and the closest surface of the lipid bilayer (blue line), $z_{s}^{(1)}$, and as a function of the of the distance between the center of the nanoparticle and the center of mass of the lipid bilayer (red), $z_{\text {com. }}$. In both cases the minimum occurs when the nanoparticle touches the membrane surface. For lower values of these distances a pocket must form within the membrane to accommodate the nanoparticle and this elastic deformation of the bilayer increases the free energy. The two snapshots show configurations of the system at the minimum, and when the nanoparticle indents the membrane and experiences a repulsive force.

ics simulations of a negatively charged lipid bilayer interacting with a positively charged nanoparticle in solution. The lipid bilayer was composed of 200 lipids and was placed in contact with an aqueous phase that contained 16000 water molecules. $25 \%$ of the lipids were DPPG (dipamitoylphosphatidylglycerol) and had a negatively charged head group while the remaining lipids had a zwitterionic head group (DPPC, dipalmitoylphosphatidylcholine). We analysed the average radial distribution function around the DPPC beads for the DPPC and DPPG beads once the simulations were equilibrated. We found the first peaks in these two distributions were at the same distance and thus concluded that the two lipids are fully mixed in the model of the membrane. The nanoparticle had a radius of $0.5 \mathrm{~nm}$ and each bead on its surface carried a positive charge, so that the total charge of the particle was +12 . 
This particle was designed to mimic a cargo with a cationic coating, which would be likely to favour binding to and permeating through the membrane. ${ }^{44}$ It should be noted that the charge of the nanoparticle is overcompensated by the negative charges on DPPG molecules so, in order to make the system charge neutral overall, $38 \mathrm{Na}^{+}$counter ions were added to the aqueous solution. We examined the distribution for the distances between each of these counter ions and the membrane surface when post processing the trajectories and found it to be uniform. We therefore concluded that the counter ions are not binding to the surface of the membrane and disrupting its structure.

Calculations were run using Gromacs $5.0^{45}$ and PLUMED 2.3. ${ }^{43,46}$ The coarse-grained Martini force field was used to represent the molecules ${ }^{47,48}$ and 16 independent replicas of the system were run for a total time of $48 \mu \mathrm{s}$. Each of these simulations was started from a different initial configurations. A 0.02 ps timestep was used to integrate the equations of motion in all calculations, together with a velocity rescale thermostat that fixed the temperature at $325 \mathrm{~K}$ and a semiisotropic Parrinello-Rahman barostat that fixed the pressure at 1 bar. We used well-tempered metadynamics ${ }^{49,50}$ and adaptive Gaussians ${ }^{51}$ with a sigma parameter of $0.5 \mathrm{~nm}$. The $\mathrm{CV}$ we biased in these simulations ran over one third of the atoms in the membrane, i.e. two atoms per lipid, and was thus a computationally-less-expensive version of equation 4 which has the sum running over all lipid atoms. The kernels in equation 1 were isotropic Gaussians with bandwidth $\lambda=3.0 \mathrm{~nm}$. Meanwhile, when solving equation 2 we used $\varphi_{0}=0.42$. For the metadynamics the hills had a height of 1.3 and were added every 100 steps. The well-tempered factor was set equal to 25. Free energy surfaces were extracted from these simulations using reweighting ${ }^{52,53}$ as we wanted to display the free energy as a function of the distance to a contour that was computed from a density field that took all the atoms in the membrane into account. The density field used to compute the CV when reweighting was thus constructed using isotropic Gaussians $(\lambda=0.6 \mathrm{~nm})$ centred on each of the lipid's atoms. This final CV was then the vertical distance, $z_{s}^{(1)}$, between the nanoparticle and an isocontour at $\varphi_{0}=7.29$. More details of the calculations together with sample input 


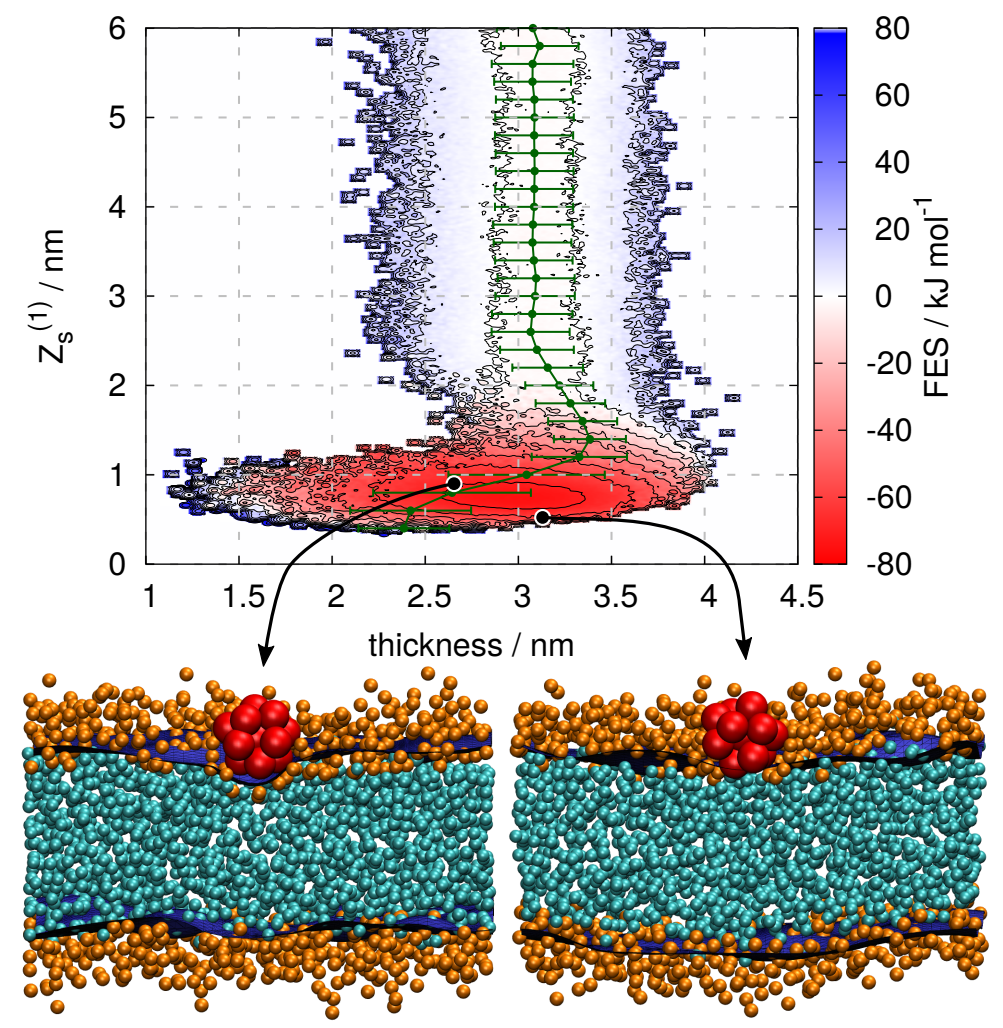

Figure 3: Contour map of the free energy computed as a function of the thickness of the bilayer under the nanoparticle and the distance between the center of the nanoparticle and the closest membrane surface, $F\left(\left|z_{s}^{(1)}-z_{s}^{(2)}\right|, z_{s}^{(1)}\right)$. The figure shows that when the nanoparticle is in solution and not interacting with the membrane $\left(z_{s}^{(1)}>2 \mathrm{~nm}\right)$, the membrane's thickness takes a constant value of $3.1 \mathrm{~nm}$. When the nanoparticle adsorbs, however, the thickness of the membrane initially increases before decreasing as the nanoparticle pushes aside the lipid molecules.

files are provided as supporting information.

Figure 2 shows the final free energy surfaces extracted from the simulations described in the previous paragraph. The blue curve shows the free energy as a function of the distance to the closer of the two surfaces, $z_{s}^{(1)}$. Meanwhile, the red curve shows the free energy as a function of the distance between the particle and membrane centres of mass, $z_{\text {com }}$. Both of these curves were obtained by reweighting the metadynamics simulations and in both cases error bars were computed using block averaging and are of the order of $1.37 \mathrm{~kJ} \mathrm{~mol}^{-1}$.

The free energy profiles in figure 2 have a shape that is typical of an adsorption process. The nanoparticle does not interact with the membrane when it is far from its surface. How- 
ever, as the particle gets closer to the membrane it begins to interact with the lipid molecules in the membrane through the electrostatic and van der Waals interactions. In addition, the process of adsorption displaces bound water molecules from the membrane surface and is thus entropically favourable. All these effects together thus ensure that there is a deep minimum in the free energy when the nanoparticle is in contact with the hydrophilic heads of the lipids. Having said that, however, as the particle indents the bilayer, the local elastic deformation of the membrane causes the free-energy to increase substantially.

The two curves in figure 2 appear on first inspection to be very similar. A closer look reveals substantial differences between the two free energy profiles, however. The standard representation, $F\left(z_{\text {com }}\right)$ (red curve), suggests a much softer and symmetric effective interaction potential than the locally-defined $F\left(z_{s}^{(1)}\right)$ (blue curve). The apparent softness of $F\left(z_{\text {com }}\right)$ at the minimum is at least partially misleading. The restoring force-constant (lower curvature) in this curve comes about because the position of the centre of mass of the membrane can easily change as the membrane moves through undulatory and peristaltic modes. It is important to note, however, that these modes are not necessarily excited by the presence of the nanoparticle. In other words, and as discussed in the introduction, when the free energy is shown as a function of $z_{\text {com }}$ there are problems in deconvolving the effect of changes in the shape of the membrane from the effects of binding. These issues are not present when the free energy is instead shown as a function of our new CV.

An additional advantage of using the new collective variable over the customary $z_{\text {com }}$ for analysis is that we can also investigate how the structure and shape of the membrane is perturbed by the nanoparticle. The centre of mass of the membrane is a collective property that depends on the position of all the lipids atoms. Consequently, for a relatively large membrane patch, its value is almost unperturbed by the adsorption of a single particle. By contrast, the distance from contour collective variable is sensitive to the local shape of the membrane, right where the nanoparticle adsorbs. Figure 3 illustrates one possible way to use such local insight. In this figure the free energy is shown as a function of the 
distance between the nanoparticle and the closer contour, $z_{s}^{(1)}$, and the distance between the contours, $\left|z_{s}^{(1)}-z_{s}^{(2)}\right|$. This latter quantity measures the thickness of the bilayer right under the particle. Clearly, nanoparticle adsorption has a substantial effect on the local thickness of the membrane. When the nanoparticle is in solution the average thickness of the membrane is $3.1 \mathrm{~nm}$. When the nanoparticle gets close to the membrane surface, however, the membrane thickness initially increases to an average value of $3.4 \mathrm{~nm}$, as the nanoparticle pulls lipid molecules out of the membrane. Subsequently, as the particle gets even closer to the surface the local thickness decreases down to $3.0 \mathrm{~nm}$ as the particle begins to enter the membrane so as to become surrounded by lipid head groups. What is more remarkable in figure 3, however, is the degree to which nanoparticle adsorption enhances the fluctuations in the local thickness of the bilayer. In other words, nanoparticle adsorption appears to excite some localized peristaltic modes.

Figure 4 further emphasizes the contrast between the insight that is provided when the $\mathrm{CV}$ defined by equation $4, s$, is used and when the reaction coordinate that is customarily used in these types of simulations, $z_{\text {com }}$ is used. Contour plots of two free energy surfaces are depicted in this figure. The lower contour map corresponds to the adsorption free energy written as a function of $z_{\text {com }}$ and the distance between the particle and the upper membrane surface, $z_{s}^{(1)}$. In the upper free energy landscape, meanwhile, $z_{s}^{(2)}$ is used in place of $z_{s}^{(1)}$. It is clear from these two surfaces that when the nanoparticle is far from the membrane all three of these collective variables are perfectly correlated. When the particle gets close to the membrane, however, there is no longer a perfect correlation between $z_{s}^{(1)}$ and the $z$ component of the distance between centers of mass, $z_{\mathrm{com}}$. As shown in the snapshots of figure 4, the correlation disappears because the adsorption of the nanoparticle locally distorts the shape of the membrane, bending its closest surface both outwards into solution or inwards into itself. As a case in point consider the snapshots shown around this figure. In three of these snapshots the distance between the nanoparticle and the upper surface, $z_{s}^{(1)}$, are almost identical. The figure shows, however, that the distances to the distal surface, $z_{s}^{(2)}$, and to the 


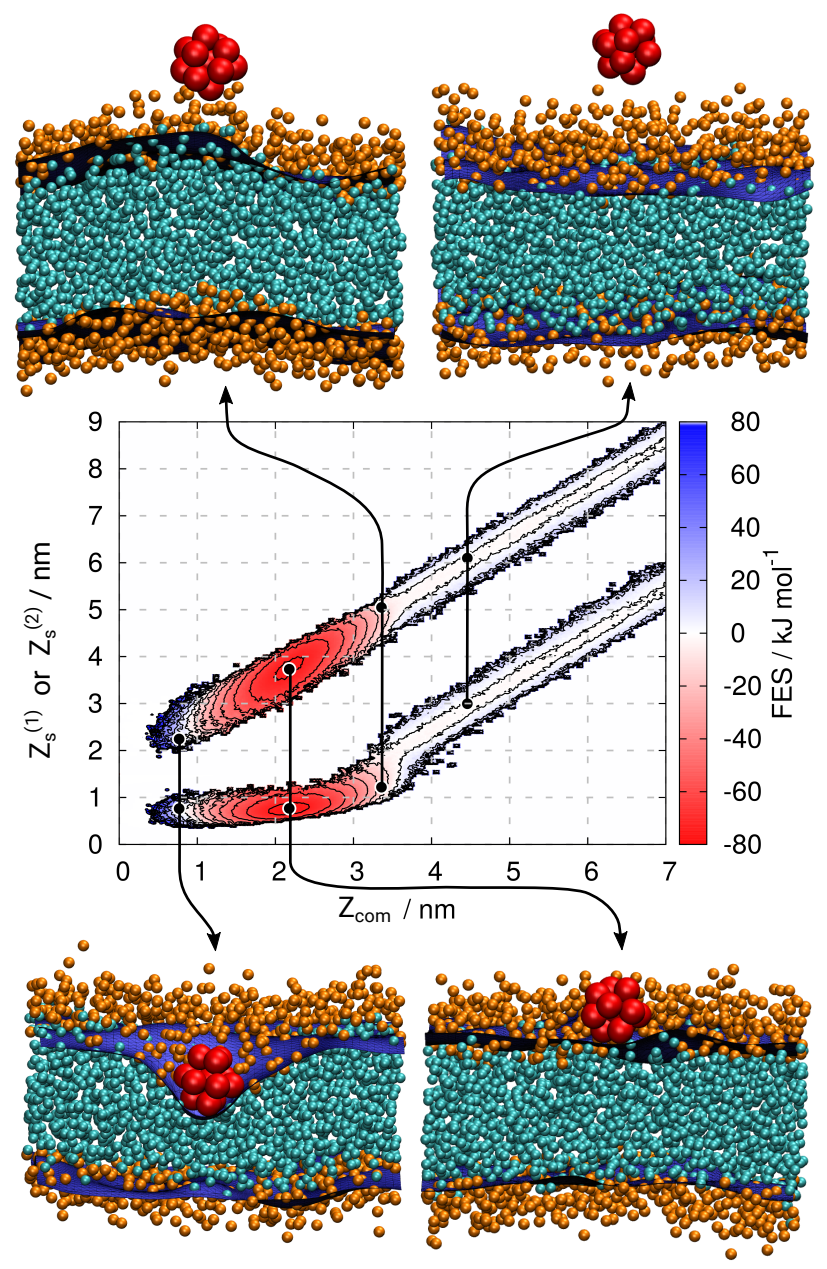

Figure 4: Contour plots of two free energy surfaces (FES). In both cases the $x$ axis measures the distance between the center of the nanoparticle and the center of mass of the membrane, $z_{\text {com }}$. For the lower FES, however, the $y$ axis measures the distance between the nanoparticle and the closest membrane surface, $z_{s}^{(1)}$. For the upper FES it measures the distance between the particle and the distal surface of the membrane, $z_{s}^{(2)}$ (see figure 1). The shape of the contour plots, and the accompanying simulation snapshots, indicate that the nanoparticle and the upper surface move up and down in a concerted manner and that this process alters the local thickness of the membrane.

center of mass of the membrane differ substantially in these three configurations. In other words, the fact that $z_{\text {com }}$ changes even though $z_{s}^{(1)}$ remains constant shows that our new CV, unlike $z_{\text {com }}$, is not affected by distortions in the shape of the upper membrane surface and long-wavelength undulations in the membrane. In addition, the fact that $z_{s}^{(2)}$ changes while $z_{s}^{(1)}$ remains constant proves that the nanoparticle's binding to the membrane has an effect on the membrane's thickness. 
To summarize we have, in this work, used well-tempered metadynamics with adaptive hills in combination with a collective variable that measures the distance from an isosurface in a density field to study the adsorption of positively charged nanoparticles into membranes. Our results show that the free energy decreases when a positively charged nanoparticle adsorbs onto a membrane that contains negatively charged lipids. Furthermore, we have shown that the new collective variable we have introduced provides insights into the mechanism of adsorption that are missed when simulations are performed using the CV that is used conventionally; namely, the distance between the nanoparticle and the centre of mass of the lipid bilayer. In particular, when we use our collective variable we are able to follow the deformations in the surface underneath the nanoparticle that occur during the adsorption process. What we observe is that as the nanoparticle moves toward the lipid membrane its closest surface initially moves outwards and towards the nanoparticle. Then, once the nanoparticle is touching the lipid bilayer and pushing on it, the surface moves inwards. These movements of the closest surface that take place during the adsorption process obviously affect the thickness of the lipid membrane by first increasing it and then decreasing it.

We have also observed that when the free energy is displayed as a function of our new $\mathrm{CV}$ the curvature at the bottom of the basin for adsorption is larger. The stiffness is larger when the distance from the membrane is used rather than the distance from centre of mass of the membrane because the distance from the intrinsic surface is less sensitive to the global fluctuations in the shape of the membrane that affect the position of the centre of mass. In other words, when it is shown as a function of the distance from contour CV that we have introduced the shape of the free energy is not affected by the physical effects such as long wavelength capillary modes or changes in volume that affect the shape of the free energy versus distance from membrane centre of mass curve.

The collective variable we have introduced could be useful when it comes to studying the process via which many solutes adsorb into lipid membranes. In particular, for membranes that exhibit ripple phases or highly deformed membranes the distance from the centre of mass 
is an inappropriate choice of reaction coordinate. Furthermore, a particularly interesting case would be the binding of curvature-sensing peptides to membranes bearing a drifting standing wave. ${ }^{54}$ More generally the freely-available and portable implementation of this $\mathrm{CV}$ in the PLUMED code will be useful to researchers studying the interaction between solutes and dynamical surfaces in other fields where these sort of approaches are already used widely.

\section{Acknowledgement}

The authors acknowledge funding from the EC-H2020-MSC-RISE-2014 programme, through project 643998 ENACT. JK, CT and GAT also acknowledge funding from EPSRC (Grants EP/P005004/1 and EP/L025124/1) while M.G.D.P acknowledges financial support from CONICET, SECTyP-UNCUYO, and FONCyT (PICT-2012-2759).

\section{Supporting Information Available}

Sample input files for PLUMED and gromacs that were used to perform and analyse the metadynamics calculations that have been described in this paper.

This material is available free of charge via the Internet at http://pubs.acs.org/.

\section{References}

(1) Wilson, D. B. Cellular transport mechanisms. Annual review of biochemistry 1978, 47, 933-965.

(2) Davis, S. J.; Ikemizu, S.; Evans, E. J.; Fugger, L.; Bakker, T. R.; van der Merwe, P. A. The nature of molecular recognition by T cells. Nature immunology 2003, 4, 217.

(3) Fulda, S.; Gorman, A. M.; Hori, O.; Samali, A. Cellular stress responses: cell survival and cell death. International journal of cell biology 2010, 2010. 
(4) Ramezanpour, M.; Leung, S.; Delgado-Magnero, K.; Bashe, B.; Thewalt, J.; Tieleman, D. Computational and experimental approaches for investigating nanoparticlebased drug delivery systems. Biochimica et Biophysica Acta (BBA)-Biomembranes 2016, 1858, 1688-1709.

(5) Yin, H.; Flynn, A. D. Drugging membrane protein interactions. Annual review of biomedical engineering 2016, 18.

(6) Jiang, Y.; Huo, S.; Mizuhara, T.; Das, R.; Lee, Y.-W.; Hou, S.; Moyano, D. F.; Duncan, B.; Liang, X.-J.; Rotello, V. M. The interplay of size and surface functionality on the cellular uptake of sub-10 nm gold nanoparticles. ACS nano 2015, 9, 9986-9993.

(7) Heikkila, E.; Martinez-Seara, H.; Gurtovenko, A. A.; Javanainen, M.; Hakkinen, H.; Vattulainen, I.; Akola, J. Cationic Au nanoparticle binding with plasma membrane-like lipid bilayers: potential mechanism for spontaneous permeation to cells revealed by atomistic simulations. The Journal of Physical Chemistry C 2014, 118, 11131-11141.

(8) Lin, J.; Zhang, H.; Chen, Z.; Zheng, Y. Penetration of lipid membranes by gold nanoparticles: insights into cellular uptake, cytotoxicity, and their relationship. ACS nano 2010, 4, 5421-5429.

(9) Zhang, S.; Gao, H.; Bao, G. Physical principles of nanoparticle cellular endocytosis. ACS nano 2015, 9, 8655-8671.

(10) Treuel, L.; Jiang, X.; Nienhaus, G. U. New views on cellular uptake and trafficking of manufactured nanoparticles. Journal of The Royal Society Interface 2013, 10, 20120939.

(11) Golchin, K.; Golchin, J.; Ghaderi, S.; Alidadiani, N.; Eslamkhah, S.; Eslamkhah, M.; Davaran, S.; Akbarzadeh, A. Gold nanoparticles applications: from artificial enzyme till drug delivery. Artificial cells, nanomedicine, and biotechnology 2018, 46, 250-254. 
(12) Fröhlich, E. The role of surface charge in cellular uptake and cytotoxicity of medical nanoparticles. International journal of nanomedicine 2012, 7, 5577.

(13) Dykman, L.; Khlebtsov, N. Gold nanoparticles in biomedical applications: recent advances and perspectives. Chemical Society Reviews 2012, 41, 2256-2282.

(14) Uddin, I.; Venkatachalam, S.; Mukhopadhyay, A.; Amil Usmani, M. Nanomaterials in the pharmaceuticals: Occurrence, behaviour and applications. Current pharmaceutical design 2016, 22, 1472-1484.

(15) Kinnear, C.; Moore, T. L.; Rodriguez-Lorenzo, L.; Rothen-Rutishauser, B.; PetriFink, A. Form Follows Function: Nanoparticle Shape and Its Implications for Nanomedicine. Chemical reviews 2017, 117, 11476-11521.

(16) Zhou, Q.; Zhang, L.; Wu, H. Nanomaterials for cancer therapies. Nanotechnology Reviews 2017, 6, 473-496.

(17) Casciola, M.; Tarek, M. A molecular insight into the electro-transfer of small molecules through electropores driven by electric fields. Biochimica et Biophysica Acta (BBA)Biomembranes 2016, 1858, 2278-2289.

(18) Shimizu, K.; Nakamura, H.; Watano, S. MD simulation study of direct permeation of a nanoparticle across the cell membrane under an external electric field. Nanoscale $\mathbf{2 0 1 6}$, 8, 11897-11906.

(19) Sun, R.; Dama, J. F.; Tan, J. S.; Rose, J. P.; Voth, G. A. Transition-tempered metadynamics is a promising tool for studying the permeation of drug-like molecules through membranes. Journal of chemical theory and computation 2016, 12, 5157-5169.

(20) Ghaemi, Z.; Minozzi, M.; Carloni, P.; Laio, A. A novel approach to the investigation of passive molecular permeation through lipid bilayers from atomistic simulations. The journal of physical chemistry B 2012, 116, 8714-8721. 
(21) Li, Y.; Yue, T.; Yang, K.; Zhang, X. Molecular modeling of the relationship between nanoparticle shape anisotropy and endocytosis kinetics. Biomaterials 2012, 33, 49654973.

(22) Quan, X.; Peng, C.; Zhao, D.; Li, L.; Fan, J.; Zhou, J. Molecular Understanding of the Penetration of Functionalized Gold Nanoparticles into Asymmetric Membranes. Langmuir 2016, 33, 361-371.

(23) Nangia, S.; Sureshkumar, R. Effects of nanoparticle charge and shape anisotropy on translocation through cell membranes. Langmuir 2012, 28, 17666-17671.

(24) da Rocha, E. L.; Caramori, G. F.; Rambo, C. R. Nanoparticle translocation through a lipid bilayer tuned by surface chemistry. Physical Chemistry Chemical Physics 2013, $15,2282-2290$.

(25) Huang, C.; Zhang, Y.; Yuan, H.; Gao, H.; Zhang, S. Role of nanoparticle geometry in endocytosis: laying down to stand up. Nano letters 2013, 13, 4546-4550.

(26) Stewart, M. P.; Sharei, A.; Ding, X.; Sahay, G.; Langer, R.; Jensen, K. F. In vitro and ex vivo strategies for intracellular delivery. Nature 2016, 538, 183.

(27) Wong-Ekkabut, J.; Baoukina, S.; Triampo, W.; Tang, I.-M.; Tieleman, D. P.; Monticelli, L. Computer simulation study of fullerene translocation through lipid membranes. Nature Nanotechnology 2008, 3, 363.

(28) Galassi, V. V.; Arantes, G. M. Partition, orientation and mobility of ubiquinones in a lipid bilayer. Biochimica et Biophysica Acta (BBA) - Bioenergetics 2015, 1847, 1560 $-1573$.

(29) Kang, M.; Loverde, S. M. Molecular simulation of the concentration-dependent interaction of hydrophobic drugs with model cellular membranes. The Journal of Physical Chemistry B 2014, 118, 11965-11972. 
(30) Gkeka, P.; Angelikopoulos, P.; Sarkisov, L.; Cournia, Z. Membrane partitioning of anionic, ligand-coated nanoparticles is accompanied by ligand snorkeling, local disordering, and cholesterol depletion. PLoS computational biology 2014, 10, e1003917.

(31) Heikkila, E.; Martinez-Seara, H.; Gurtovenko, A. A.; Javanainen, M.; Hakkinen, H.; Vattulainen, I.; Akola, J. Cationic Au nanoparticle binding with plasma membrane-like lipid bilayers: potential mechanism for spontaneous permeation to cells revealed by atomistic simulations. The Journal of Physical Chemistry C 2014, 118, 11131-11141.

(32) Neale, C.; Pomès, R. Sampling errors in free energy simulations of small molecules in lipid bilayers. Biochimica et Biophysica Acta (BBA)-Biomembranes 2016, 1858, 25392548.

(33) Neale, C.; Bennett, W. D.; Tieleman, D. P.; Pomès, R. Statistical convergence of equilibrium properties in simulations of molecular solutes embedded in lipid bilayers. Journal of Chemical Theory and Computation 2011, 7, 4175-4188.

(34) Filipe, H. A.; Moreno, M. J.; Rog, T.; Vattulainen, I.; Loura, L. M. How to tackle the issues in free energy simulations of long amphiphiles interacting with lipid membranes: convergence and local membrane deformations. The Journal of Physical Chemistry B 2014, 118, 3572-3581.

(35) Shinoda, W. Permeability across lipid membranes. Biochimica et Biophysica Acta (BBA) - Biomembranes 2016, 1858, 2254 - 2265, Biosimulations of lipid membranes coupled to experiments.

(36) Kopelevich, D. I. One-dimensional potential of mean force underestimates activation barrier for transport across flexible lipid membranes. The Journal of chemical physics 2013, 139, 10B602_1.

(37) Willard, A. P.; Chandler, D. Instantaneous liquid interfaces. The Journal of Physical Chemistry B 2010, 114, 1954-1958. 
(38) Varilly, P.; Chandler, D. Water evaporation: A transition path sampling study. The Journal of Physical Chemistry B 2013, 117, 1419-1428.

(39) Darvas, M.; Jorge, M.; Cordeiro, M. N. D. S.; Kantorovich, S. S.; Sega, M.; Jedlovszky, P. Calculation of the Intrinsic Solvation Free Energy Profile of an Ionic Penetrant Across a Liquid-Liquid Interface with Computer Simulations. J. Phys. Chem. B 2013, 117, 16148-16156.

(40) Tarazona, P.; Chacón, E.; Bresme, F. Thermal fluctuations and bending rigidity of bilayer membranes. 2013, 139, 094902-15.

(41) Loubet, B.; Lomholt, M. A.; Khandelia, H. Tension moderation and fluctuation spectrum in simulated lipid membranes under an applied electric potential. 2013, 139, 164902.

(42) Chacn, E.; Tarazona, P.; Bresme, F. A computer simulation approach to quantify the true area and true area compressibility modulus of biological membranes. The Journal of Chemical Physics 2015, 143, 034706.

(43) Tribello, G. A.; Bonomi, M.; Branduardi, D.; Camilloni, C.; Bussi, G. PLUMED 2 : New feathers for an old bird. Computer Physics Communications 2014, 185, 604-613.

(44) Lin, J.; Alexander-Katz, A. Cell Membranes Open "Doors" for Cationic Nanoparticles/Biomolecules: Insights into Uptake Kinetics. ACS Nano 2013, 7, 10799-10808.

(45) Abraham, M. J.; Murtola, T.; Schulz, R.; Páll, S.; Smith, J. C.; Hess, B.; Lindahl, E. GROMACS: High performance molecular simulations through multi-level parallelism from laptops to supercomputers. SoftwareX 2015, 1, 19-25.

(46) Baldi, E.; Ceriotti, M.; Tribello, G. A. Extracting the interfacial free energy and anisotropy from a smooth fluctuating dividing surface. Journal of Physics: Condensed Matter 2017, 29, 445001. 
(47) Marrink, S. J.; Risselada, H. J.; Yefimov, S.; Tieleman, D. P.; De Vries, A. H. The MARTINI force field: coarse grained model for biomolecular simulations. The journal of physical chemistry B 2007, 111, 7812-7824.

(48) Marrink, S. J.; De Vries, A. H.; Mark, A. E. Coarse grained model for semiquantitative lipid simulations. The Journal of Physical Chemistry B 2004, 108, 750-760.

(49) Laio, A.; Parrinello, M. Escaping free-energy minima. Proceedings of the National Academy of Sciences 2002, 99, 12562-12566.

(50) Barducci, A.; Bussi, G.; Parrinello, M. Well-tempered metadynamics: a smoothly converging and tunable free-energy method. Physical review letters 2008, 100, 020603.

(51) Branduardi, D.; Bussi, G.; Parrinello, M. Metadynamics with adaptive Gaussians. Journal of chemical theory and computation 2012, 8, 2247-2254.

(52) Bonomi, M.; Barducci, A.; Parrinello, M. Reconstructing the equilibrium Boltzmann distribution from well-tempered metadynamics. Journal of computational chemistry 2009, 30, 1615-1621.

(53) Tiwary, P.; Parrinello, M. A time-independent free energy estimator for metadynamics. The Journal of Physical Chemistry B 2014, 119, 736-742.

(54) Gómez-Llobregat, J.; Elías-Wolff, F.; Lindén, M. Anisotropic Membrane Curvature Sensing by Amphipathic Peptides. Biophysj 2016, 110, 197-204. 